\title{
On the flexoelectric deformations of finite size bodies
}

\author{
A. S. Yurkov \\ 644076, Omsk, Russia,e-mail: fitec@mail.ru
}

(Dated: April 6, 2019)

\begin{abstract}
Exact equations describing flexoelectric deformation in solids, derived previously within the framework of a continuum media theory, are partial differential equations of the fourth order. They are too complex to be used in the cases interesting for applications. In this paper, using the fact of smallness of the elastic moduli of a higher order, simplified equations are proposed. Solution of the exact equations is approximately represented as a sum of two parts: the first part obeys onedimensional differential equations and exponentially decays near surface, the second - obeys the equations of classical theory of elasticity. The first part can be constructed in an explicit form. For the second part, boundary conditions are obtained. They have a form of the classical boundary conditions for the body under external forces on surface.

PACS numbers: 77.22.-d, 77.65.-j, 77.90.+k
\end{abstract}

\section{INTRODUCTION}

Although flexoelectric effect has been known for a long time (see $\frac{1.2}{2}$ and references therein), it is still the subject of intensive studies ( $\mathrm{see}^{3, \underline{4}}$ and references therein). In the practical sense the importance of flexoelectricity is that it, in contrast to piezoelectricity, is symmetrically allowed in centro-symmetric materials and therefore broadens the choice of materials which can be used for electromechanical devices. Besides, although flexoelectricity is weak effect at macroscale, at nanoscale it becomes much stronger: reduced dimensions imply large gradients. From a theoretical point of view it is interesting that in the case of the flexoelectric effect there is non-trivial relation between the direct and converse effect. There is a certain asymmetry in the bulk constitutive electromechanical equations: while linearly varying the strain obviously contributes to the homogeneous part of the polarization, the homogeneous polarization does not appear in the equations that define the elastic stresses. Based on this, some authors have concluded that the flexoelectric effect, at least for a special arrangement, has no reversibility (see references in 5 ). But the general principles of thermodynamics require the effect to be reversible. This paradox is resolved as follows 5.6 . The equations of elastic equilibrium are differential, so they should be appended by boundary conditions. In the presence of the flexoelectric effect, the elastic boundary conditions have non-classical form, and they include not only the gradients of polarization, but also the polarization itself ${ }^{6}$. This fact leads to distortion of real body of a finite size by homogeneous polarization.

However, it is worth mentioning that in the presence of flexoelectricity not only the elastic boundary conditions should be changed, but also the bulk differential equations of elasticity equilibrium should be modified 6 . The exact (within the framework of a continuum media theory) equations are partial differential equations of the fourth order, and they are too complex to be used in the cases interesting for applications. The only case when an analytical solution is passable is a ball of an isotropic dielectric ${ }^{7}$. Even for such a simple geometry the solution is cumbersome and requires an introduction of non-standard special functions. This is why the development of methods of approximate description of flexoelectric deformations is interesting. To develop one of such methods is a subject of the present short note.

\section{DECOMPOSITION OF ELASTIC DISPLACEMENTS TO CLASSICAL AND NON-CLASSICAL PARTS AND EXPLICIT REPRESENTATION OF NON-CLASSICAL PART}

$\mathrm{In}^{6}$ equations of elastic equilibrium and the corresponding boundary conditions were derived using the Cartesian coordinate system. However, for certain problems the curvilinear coordinates are more appropriate. Equations in curvilinear coordinates were derived in ${ }^{7}$. In such coordinates the differential equations of elastic equilibrium are

$$
c^{\alpha \beta \gamma \delta} u_{\gamma ; \delta ; \beta}+f^{\gamma \delta \alpha \beta} P_{\gamma ; \delta ; \beta}-v^{\alpha \beta \gamma \delta \varepsilon \zeta} u_{\gamma ; \delta ; \zeta ; \varepsilon ; \beta}=0
$$

where the lower Greek indices separated by semicolons denote covariant derivatives. The usual partial derivatives will be denoted by lower Greek indices separated by comma. For other notations see 7 .

The boundary conditions for these equations in the coordinate system where the equation of the body surface is $x^{3}=x_{S}^{3}=$ const may have different equivalent forms. Here we assume the following form

$$
\Theta^{\alpha 33}=0
$$




$$
\sigma^{\alpha 3}-\Theta_{; \beta}^{\alpha \beta 3}+\Theta_{, 3}^{\alpha 33}+\Theta_{(\beta \gamma)}^{\alpha(\beta \gamma)} \Gamma_{(\beta \gamma}^{3}=0
$$

where

$$
\begin{gathered}
\sigma^{\alpha \beta}=c^{\alpha \beta \gamma \delta} u_{\gamma ; \delta}+f^{\gamma \delta \alpha \beta} P_{\gamma ; \delta}-v^{\alpha \beta \gamma \delta \varepsilon \zeta} u_{\gamma ; \delta ; \zeta ; \varepsilon}, \\
\Theta^{\alpha \beta \gamma}=v^{\alpha \beta \varepsilon \delta \gamma \zeta} u_{\varepsilon ; \delta ; \zeta}-\frac{1}{2} f^{\delta \gamma \alpha \beta} P_{\delta} .
\end{gathered}
$$

The indices enclosed in parentheses runs only the values 1 and 2 , if there are multiple indexes in one pair of parentheses then these indices are not equal to 3 simultaneously. Actually the equations (2) are equations (11), and (3) are slightly transformed equations (12) from ${ }^{7}$.

When solving the problem for a ball ${ }^{7}$ we have seen that the solution is a sum of two parts. The first part is a solution of the equations of the classical theory of elasticity, the second is a non-classical part which for small $v^{\alpha \beta \gamma \delta \varepsilon \zeta}$ is concentrated near the surface and decays exponentially inside the body. Hereafter we call the first one a volume, or a classical part, and the second one a non-classical, or a surface part. It is naturally to assume that this property should be preserved in more general cases. Our aim is to use this observation for construction of approximation that is useful for solution of more general problems than the case of a ball.

According to the observation mentioned above we present the solution of the equation (11) in the form $u_{\gamma}=\tilde{u}_{\gamma}+\hat{u}_{\gamma}$, where $\tilde{u}_{\gamma}$ obey the classical equations:

$$
c^{\alpha \beta \gamma \delta} \tilde{u}_{\gamma ; \delta ; \beta}+f^{\gamma \delta \alpha \beta} P_{\gamma ; \delta ; \beta}=0 .
$$

Using (6) and (11) we get that $\hat{u}_{\gamma}$ obey differential equations:

$$
c^{\alpha \beta \gamma \delta} \hat{u}_{\gamma ; \delta ; \beta}-v^{\alpha \beta \gamma \delta \varepsilon \zeta} \hat{u}_{\gamma ; \delta ; \zeta ; \varepsilon ; \beta}=v^{\alpha \beta \gamma \delta \varepsilon \zeta} \tilde{u}_{\gamma ; \delta ; \zeta ; \varepsilon ; \beta} .
$$

Substitution $u_{\gamma}=\tilde{u}_{\gamma}+\hat{u}_{\gamma}$ and some transformations also yields that the boundary conditions (2) and (3) takes the form:

$$
\begin{gathered}
v^{\alpha 3 \varepsilon \delta 3 \zeta} \hat{u}_{\varepsilon ; \delta ; \zeta}=\frac{1}{2} f^{\delta 3 \alpha 3} P_{\delta}-v^{\alpha 3 \varepsilon \delta 3 \zeta} \tilde{u}_{\varepsilon ; \delta ; \zeta}, \\
c^{\alpha 3 \gamma \delta} \tilde{u}_{\gamma ; \delta}+f^{\gamma \delta \alpha 3} P_{\gamma ; \delta}-v^{\alpha 3 \gamma \delta \varepsilon \zeta} \tilde{u}_{\gamma ; \delta ; \zeta ; \varepsilon}-v^{\alpha(\beta) \varepsilon \delta 3 \zeta} \tilde{u}_{\varepsilon ; \delta ; \zeta ;(\beta)}+\frac{1}{2} f^{\delta 3 \alpha(\beta)} P_{\delta ;(\beta)}+v^{\alpha(\beta) \varepsilon \delta(\gamma) \zeta} \tilde{u}_{\varepsilon ; \delta ; \zeta} \Gamma_{(\beta)(\gamma)}^{3}- \\
\frac{1}{2} f^{\delta(\gamma) \alpha(\beta)} P_{\delta} \Gamma_{(\beta)(\gamma)}^{3}+c^{\alpha 3 \gamma \delta} \hat{u}_{\gamma ; \delta}-v^{\alpha 3 \gamma \delta \varepsilon \zeta} \hat{u}_{\gamma ; \delta ; \zeta ; \varepsilon}-v^{\alpha(\beta) \varepsilon \delta 3 \zeta} \hat{u}_{\varepsilon ; \delta ; \zeta ;(\beta)}+v^{\alpha(\beta) \varepsilon \delta(\gamma) \zeta} \hat{u}_{\varepsilon ; \delta ; \zeta} \Gamma_{(\beta)(\gamma)}^{3}=0
\end{gathered}
$$

Naturally decomposition $u_{\gamma}=\tilde{u}_{\gamma}+\hat{u}_{\gamma}$ is not unique. By requiring that $\tilde{u}_{\gamma}$ obey (6), we have restricted this non-uniqueness, but have not eliminated it completely. It is clear that $\tilde{u}_{\gamma}$ does not contain a non-classical part, but it does not mean that $\hat{u}_{\gamma}$ does not contain a classical part. Thus further restriction is required. This restriction will be done in the following way: when constructing the explicit form of $\hat{u}_{\gamma}$, we will discard the non-exponential terms. The details will be clear from what follows.

Below we use the fact that real values of $v^{\alpha \beta \gamma \delta \varepsilon \zeta}$ are small. It is convenient to assume that all of them are proportional to a scalar $v \rightarrow 0$. We assume also that all $c^{\alpha \beta \gamma \delta}$ is proportional to a scalar $c$. If $v \rightarrow 0$ then the right-hand side of (7) and the second term on the right hand side of (8) can be neglected. Indeed since $\tilde{u}_{\gamma}$ obey the classical equation (6) in the case of sufficiently smooth surface and in the absence of polarization gradients tend to infinity in the limit $v \rightarrow 0$, the classical part can not have large gradients which can compensate smallness of $v^{\alpha \beta \gamma \delta \varepsilon \zeta}$. As for the non-classical part $\hat{u}_{\gamma}$, the situation is different: here such compensation is possible, but only if the derivatives are in $x^{3}$. Moreover, in a thin layer near the surface covariant derivatives in $x^{3}$ can be replaced by the usual derivatives, $\Gamma$-terms give only small corrections here. Thus (7) and (8) can be approximately replaced by

$$
\begin{gathered}
c^{\alpha 3 \gamma 3} \hat{u}_{\gamma, 3,3}-v^{\alpha 3 \gamma 333} \hat{u}_{\gamma, 3,3,3,3}=0, \\
v^{\alpha 3 \varepsilon 333} \hat{u}_{\varepsilon, 3,3}=\frac{1}{2} f^{\delta 3 \alpha 3} P_{\delta} .
\end{gathered}
$$


Equations (10) are a system of three ordinal differential equations, the dependence on $x^{1}$ and $x^{2}$ is parametric here. Moreover, in a thin layer near the body surface one can assume that the coefficients do not depends on $x^{3}$, they are approximately equal to the surface values. Solution of such a system is easy. In a standard way one should find a fundamental basis set of solutions in the form $\hat{u}_{\gamma}=\bar{u}_{\gamma} e^{\lambda\left(x^{3}-x_{S}^{3}\right)}$. Obviously equations for amplitudes $\bar{u}_{\gamma}$ are

$$
\lambda^{2} c^{\alpha 3 \gamma 3} \bar{u}_{\gamma}=\lambda^{4} v^{\alpha 3 \gamma 333} \bar{u}_{\gamma} .
$$

It is clear from (12) that fundamental solutions with $\lambda=0$ are possible. But it is clear also that such $\lambda$ corresponds to non-exponential, classical solutions. This is why we should exclude the case of $\lambda=0$. By this exclusion we completely eliminate the non-uniqueness of the decomposition $u_{\gamma}=\tilde{u}_{\gamma}+\hat{u}_{\gamma}$ mentioned above. Thus (12) should be changed to

$$
c^{\alpha 3 \gamma 3} \bar{u}_{\gamma}=\lambda^{2} v^{\alpha 3 \gamma 333} \bar{u}_{\gamma} .
$$

Equation (13) is a standard generalized eigenproblem for symmetric positive definite $3 \times 3$ matrices. Therefore we consider $\lambda_{n}$ and $\bar{u}_{\gamma}^{n}$ to be known. If the body bulk corresponds to $x^{3} \leq x_{S}^{3}$ for definiteness, then $\lambda_{n}$ equals the positive square root of the $n$-th eigenvalue. General representation of non-classical part is

$$
\hat{u}_{\gamma}=\sum_{n=1}^{3} a_{n} \bar{u}_{\gamma}^{n} e^{\lambda_{n}\left(x^{3}-x_{S}^{3}\right)},
$$

where only the coefficients $a_{n}$ are unknown. The latter can be found easily by (11) which yields a simple system of linear algebraic equations:

$$
\sum_{n=1}^{3} \lambda_{n}^{2} v^{\alpha 3 \gamma 333} \bar{u}_{\gamma}^{n} a_{n}=\frac{1}{2} f^{\delta 3 \alpha 3} P_{\delta} .
$$

Note that the smallness of $v^{\alpha 3 \gamma 333}$ in (15) is compensated by $\lambda_{n}^{2}$ and using (13) this system of equations can be rewritten in the form:

$$
\sum_{n=1}^{3} c^{\alpha 3 \gamma 3} \bar{u}_{\gamma}^{n} a_{n}=\frac{1}{2} f^{\delta 3 \alpha 3} P_{\delta} .
$$

Thus the non-classical part of elastic displacements is found completely in explicit form.

\section{BOUNDARY CONDITIONS FOR THE CLASSICAL PART}

We saw above that to find completely the non-classical part of elastic displacements one needs only the boundary conditions (8). The remaining boundary conditions (9) yield the boundary conditions for the classical equations (6) in this way, one should only substitute known $\hat{u}_{\gamma}$ to (9). But to avoid the excess of accuracy, all the terms which tend to zero in the limit of $v \rightarrow 0$ should be omitted in the final form of these boundary conditions. Besides, further down it will be clear that (9) contains terms which diverge in this limit. However it is worth mentioning that these divergent terms completely cancel each other out. So these terms should be omitted.

According to the above, all the terms in (9) where gradients of $\tilde{u}_{\gamma}$ are convolved with $v^{\alpha \beta \gamma \delta \varepsilon \zeta}$ should be omitted for the same reasons as in the case of the equation (77). Further it is convenient to introduce the notation:

$$
s^{\alpha}=-c^{\alpha 3 \gamma \delta} \hat{u}_{\gamma ; \delta}+v^{\alpha 3 \gamma \delta \varepsilon \zeta} \hat{u}_{\gamma ; \delta ; \zeta ; \varepsilon}+v^{\alpha(\beta) \gamma \delta 3 \zeta} \hat{u}_{\gamma ; \delta ; \zeta ;(\beta)}-v^{\alpha(\beta) \varepsilon \delta(\gamma) \zeta} \hat{u}_{\varepsilon ; \delta ; \zeta} \Gamma_{(\beta)(\gamma)}^{3} .
$$

With this notation the boundary conditions to the equations of classic theory of elasticity can be written as

$$
c^{\alpha 3 \gamma \delta} \tilde{u}_{\gamma ; \delta}=s^{\alpha}-f^{\gamma \delta \alpha 3} P_{\gamma ; \delta}-\frac{1}{2} f^{\delta 3 \alpha(\beta)} P_{\delta ;(\beta)}+\frac{1}{2} f^{\delta(\gamma) \alpha(\beta)} P_{\delta} \Gamma_{(\beta)(\gamma)}^{3} .
$$

Now all the terms to be further simplified, are contained in $s^{\alpha}$. Carrying out simplification, first of all one should leave in the last term only the partial (not covariant) derivatives in $x^{3}$. The reason for this is the same as above. Next, one should express the remaining covariant derivatives in terms of partial derivatives. The exact expression for the third covariant derivative of the vector is very cumbersome. But keeping in mind that these third covariant 
derivatives are convolved with $v^{\alpha \beta \gamma \delta \varepsilon \zeta}$, one can omit all the terms where initial vector is not differentiated at least two times. So it turns out:

$$
A_{\alpha ; \beta ; \gamma ; \zeta} \approx A_{\alpha, \beta, \gamma, \zeta}-A_{\delta, \beta, \zeta} \Gamma_{\alpha \gamma}^{\delta}-A_{\delta, \gamma, \zeta} \Gamma_{\alpha \beta}^{\delta}-A_{\delta, \beta, \gamma} \Gamma_{\alpha \zeta}^{\delta}-A_{\alpha, \delta, \zeta} \Gamma_{\beta \gamma}^{\delta}-A_{\alpha, \delta, \gamma} \Gamma_{\beta \zeta}^{\delta}-A_{\alpha, \beta, \delta} \Gamma_{\gamma \zeta}^{\delta}
$$

With this equation and simplifications mentioned above it turns

$$
\begin{aligned}
& s^{\alpha}= \\
& -c^{\alpha 3 \gamma \delta} \hat{u}_{\gamma ; \delta}+v^{\alpha 3 \gamma \delta \varepsilon \zeta} \hat{u}_{\gamma, \delta, \zeta, \varepsilon}-v^{\alpha 3 \gamma \delta \varepsilon \zeta} \hat{u}_{\rho, \delta, \varepsilon} \Gamma_{\gamma \zeta}^{\rho}-v^{\alpha 3 \gamma \delta \varepsilon \zeta} \hat{u}_{\rho, \zeta, \varepsilon} \Gamma_{\gamma \delta}^{\rho}-v^{\alpha 3 \gamma \delta \varepsilon \zeta} \hat{u}_{\rho, \delta, \zeta} \Gamma_{\gamma \varepsilon}^{\rho}-v^{\alpha 3 \gamma \delta \varepsilon \zeta} \hat{u}_{\gamma, \rho, \varepsilon} \Gamma_{\delta \zeta}^{\rho}- \\
& v^{\alpha 3 \gamma \delta \varepsilon \zeta} \hat{u}_{\gamma, \rho, \zeta} \Gamma_{\delta \varepsilon}^{\rho}-v^{\alpha 3 \gamma \delta \varepsilon \zeta} \hat{u}_{\gamma, \delta, \rho} \Gamma_{\zeta \varepsilon}^{\rho}+v^{\alpha(\beta) \gamma \delta 3 \zeta} \hat{u}_{\gamma, \delta, \zeta,(\beta)}-v^{\alpha(\beta) \gamma \delta 3 \zeta} \hat{u}_{\rho, \delta,(\beta)} \Gamma_{\gamma \zeta}^{\rho}-v^{\alpha(\beta) \gamma \delta 3 \zeta} \hat{u}_{\rho, \zeta,(\beta)} \Gamma_{\gamma \delta}^{\rho}- \\
& v^{\alpha(\beta) \gamma \delta 3 \zeta} \hat{u}_{\rho, \delta, \zeta} \Gamma_{\gamma(\beta)}^{\rho}-v^{\alpha(\beta) \gamma \delta 3 \zeta} \hat{u}_{\gamma, \rho,(\beta)} \Gamma_{\delta \zeta}^{\rho}-v^{\alpha(\beta) \gamma \delta 3 \zeta} \hat{u}_{\gamma, \rho, \zeta} \Gamma_{\delta(\beta)}^{\rho}-v^{\alpha(\beta) \gamma \delta 3 \zeta} \hat{u}_{\gamma, \delta, \rho} \Gamma_{\zeta(\beta)}^{\rho}- \\
& v^{\alpha(\beta) \varepsilon 3(\gamma) 3} \hat{u}_{\varepsilon, 3,3} \Gamma_{(\beta)(\gamma)}^{3} .
\end{aligned}
$$

Again, using the fact that to compensate smallness of $v^{\alpha \beta \gamma \delta \varepsilon \zeta}$ one needs at least two derivatives in $x^{3}$, we further simplify this equation:

$$
\begin{aligned}
& s^{\alpha}= \\
& -c^{\alpha 3 \gamma \delta} \hat{u}_{\gamma ; \delta}+v^{\alpha 3 \gamma \delta \varepsilon \zeta} \hat{u}_{\gamma, \delta, \zeta, \varepsilon}-v^{\alpha 3 \gamma 33 \zeta} \hat{u}_{\rho, 3,3} \Gamma_{\gamma \zeta}^{\rho}-v^{\alpha 3 \gamma \delta 33} \hat{u}_{\rho, 3,3} \Gamma_{\gamma \delta}^{\rho}-v^{\alpha 3 \gamma 3 \varepsilon 3} \hat{u}_{\rho, 3,3} \Gamma_{\gamma \varepsilon}^{\rho}-v^{\alpha 3 \gamma \delta 3 \zeta} \hat{u}_{\gamma, 3,3} \Gamma_{\delta \zeta}^{3}- \\
& v^{\alpha 3 \gamma \delta \varepsilon 3} \hat{u}_{\gamma, 3,3} \Gamma_{\delta \varepsilon}^{3}-v^{\alpha 3 \gamma 3 \varepsilon \zeta} \hat{u}_{\gamma, 3,3} \Gamma_{\zeta \varepsilon}^{3}+v^{\alpha(\beta) \gamma 333} \hat{u}_{\gamma, 3,3,(\beta)}-v^{\alpha(\beta) \gamma 333} \hat{u}_{\rho, 3,3} \Gamma_{\gamma(\beta)}^{\rho}-v^{\alpha(\beta) \gamma \delta 33} \hat{u}_{\gamma, 3,3} \Gamma_{\delta(\beta)}^{3}- \\
& v^{\alpha(\beta) \gamma 33 \zeta} \hat{u}_{\gamma, 3,3} \Gamma_{\zeta(\beta)}^{3}-v^{\alpha(\beta) \varepsilon 3(\gamma) 3} \hat{u}_{\varepsilon, 3,3} \Gamma_{(\beta)(\gamma)}^{3} .
\end{aligned}
$$

It is convenient to introduce yet another notation:

$$
\begin{aligned}
& h^{\alpha \rho}=v^{\alpha 3 \gamma 33 \zeta} \Gamma_{\gamma \zeta}^{\rho}+v^{\alpha 3 \gamma \delta 33} \Gamma_{\gamma \delta}^{\rho}+v^{\alpha 3 \gamma 3 \varepsilon 3} \Gamma_{\gamma \varepsilon}^{\rho}+v^{\alpha 3 \rho \delta 3 \zeta} \Gamma_{\delta \zeta}^{3}+v^{\alpha 3 \rho \delta \varepsilon 3} \Gamma_{\delta \varepsilon}^{3}+v^{\alpha 3 \rho 3 \varepsilon \zeta} \Gamma_{\zeta \varepsilon}^{3}+v^{\alpha(\beta) \gamma 333} \Gamma_{\gamma(\beta)}^{\rho}+ \\
& v^{\alpha(\beta) \rho \delta 33} \Gamma_{\delta(\beta)}^{3}+v^{\alpha(\beta) \rho 33 \zeta} \Gamma_{\zeta(\beta)}^{3}+v^{\alpha(\beta) \rho 3(\gamma) 3} \Gamma_{(\beta)(\gamma)}^{3} .
\end{aligned}
$$

Using the symmetry properties of $v^{\alpha \beta \gamma \delta \varepsilon \zeta}$, it can be rewritten as:

$$
h^{\alpha \beta}=\left(v^{\alpha 3 \gamma 3 \delta 3}+2 v^{\alpha 3 \gamma \delta 33}\right) \Gamma_{\gamma \delta}^{\beta}+\left(2 v^{\alpha 3 \beta \gamma \delta 3}+v^{\alpha 3 \beta \gamma 3 \delta}\right) \Gamma_{\gamma \delta}^{3}+2 v^{\alpha 3 \beta \gamma(\delta) 3} \Gamma_{\gamma(\delta)}^{3}+v^{\alpha 3 \gamma 3(\delta) 3} \Gamma_{\gamma(\delta)}^{\beta}+v^{\alpha(\gamma) \beta 3(\delta) 3} \Gamma_{(\gamma)(\delta)}^{3} .
$$

In this notation the expression for $s^{\alpha}$ can be written as follows:

$$
s^{\alpha}=-c^{\alpha 3 \gamma \delta} \hat{u}_{\gamma, \delta}+c^{\alpha 3 \gamma \delta} \hat{u}_{\varepsilon} \Gamma_{\gamma \delta}^{\varepsilon}+v^{\alpha 3 \gamma \delta \varepsilon \zeta} \hat{u}_{\gamma, \delta, \zeta, \varepsilon}+v^{\alpha(\beta) \gamma 333} \hat{u}_{\gamma, 3,3,(\beta)}-h^{\alpha \beta} \hat{u}_{\beta, 3,3} .
$$

Now one can proceed to the analysis of the divergent terms. Obviously they appear in the first and third term, when all the derivatives here are in $x^{3}$. But it is also clear that these divergent terms exactly cancel each other out because of the explicit form of $\hat{u}_{\gamma}$, derived above. One might think that these divergent terms should simply be excluded, but in reality the situation is more complicated.

The fact is that $-c^{\alpha 3 \gamma 3} \hat{u}_{\gamma, 3,3}+v^{\alpha 3 \gamma 333} \hat{u}_{\gamma, 3,3,3,3}$ is equal to zero only for the main approximation for $\hat{u}_{\gamma}$. But the corrections to this approximation may result in the fact that this expression becomes finite. Henceforth we denote such corrections as $w_{\gamma}$ keeping notation $\hat{u}_{\gamma}$ only for the main approximation. In this notation we can write:

$$
s^{\alpha}=-c^{\alpha 3 \gamma 3} w_{\gamma, 3}+v^{\alpha 3 \gamma 333} w_{\gamma, 3,3,3}-c^{\alpha 3 \gamma(\delta)} \hat{u}_{\gamma,(\delta)}+c^{\alpha 3 \gamma \delta} \hat{u}_{\varepsilon} \Gamma_{\gamma \delta}^{\varepsilon}+v^{\alpha 3 \gamma \delta \varepsilon \zeta} \hat{u}_{\gamma(, \delta, \zeta, \varepsilon)}+v^{\alpha(\beta) \gamma 333} \hat{u}_{\gamma, 3,3,(\beta)}-h^{\alpha \beta} \hat{u}_{\beta, 3,3} .
$$

To compensate the smallness of $v^{\alpha \beta \gamma \delta \varepsilon \zeta}$ in the fifth term of this equation there should be at least two differentiations in $x^{3}$. Thus after some reductions one can rewrite (25) as follows:

$$
s^{\alpha}=-c^{\alpha 3 \gamma 3} w_{\gamma, 3}+v^{\alpha 3 \gamma 333} w_{\gamma, 3,3,3}-c^{\alpha 3 \gamma(\delta)} \hat{u}_{\gamma,(\delta)}+c^{\alpha 3 \gamma \delta} \hat{u}_{\varepsilon} \Gamma_{\gamma \delta}^{\varepsilon}+2\left(v^{\alpha 3 \gamma(\beta) 33}+v^{\alpha 3 \gamma 3(\beta) 3}\right) \hat{u}_{\gamma, 3,3,(\beta)}-h^{\alpha \beta} \hat{u}_{\beta, 3,3} .
$$

It is clear that in (26) one should use only corrections $w_{\gamma}$ of order $v^{1 / 2}$. Only such corrections yield a contribution of the same order as the other terms. To find the corresponding contributions, we should keep in the exact equation

$$
c^{\alpha \beta \gamma \delta}\left(\hat{u}_{\gamma}+w_{\gamma}\right)_{; \delta ; \beta}-v^{\alpha \beta \gamma \delta \varepsilon \zeta}\left(\hat{u}_{\gamma}+w_{\gamma}\right)_{; \delta ; \zeta ; \varepsilon ; \beta}=v^{\alpha \beta \gamma \delta \varepsilon \zeta} \tilde{u}_{\gamma ; \delta ; \zeta ; \varepsilon ; \beta}
$$


only the terms of the order $v^{-1 / 2}$. Indeed if $w_{\gamma} \sim v^{1 / 2}$ then the main terms $w_{\gamma, 3,3}$ and $v^{\alpha 3 \gamma 333} w_{\gamma, 3,3,3,3}$ have just such an order. Note that although there are terms $\hat{u}_{\gamma, 3,3}$ and $v^{\alpha 3 \gamma 333} \hat{u}_{\gamma, 3,3,3,3}$ in the equation, which are of the order $\sim v^{-1}$, now they cancel each other completely due to the fact that now $\hat{u}_{\gamma}$ denotes the main approximation. Right hand side of (27) is $\sim v$, so it should be omitted. The terms $c^{\alpha \beta \gamma \delta} w_{\gamma(; \delta ; \beta)}$ and $v^{\alpha \beta \gamma \delta \varepsilon \zeta} w_{\gamma(; \delta ; \zeta ; \varepsilon ; \beta)}$ should be omitted by the same reasons. Thus (27) is reduced to

$$
-c^{\alpha 3 \gamma 3} w_{\gamma, 3,3}+v^{\alpha 3 \gamma 333} w_{\gamma, 3,3,3,3}=c^{\alpha \beta \gamma \delta} \hat{u}_{\gamma(; \delta ; \beta)}-v^{\alpha \beta \gamma \delta \varepsilon \zeta} \hat{u}_{\gamma(; \delta ; \zeta ; \varepsilon ; \beta)} .
$$

Equation (28) requires further simplification: in the right-hand side we should keep only the terms $\sim v^{-1 / 2}$. For this in the first term there should be one partial derivative in $x^{3}$, and in the second term there should be three derivatives in $x^{3}$. This is why we simplify second and fourth covariant derivatives as:

$$
\begin{gathered}
\hat{u}_{\gamma ; \delta ; \beta} \approx \hat{u}_{\gamma, \delta, \beta}-\hat{u}_{\gamma, \rho} \Gamma_{\delta \beta}^{\rho}-\hat{u}_{\rho, \delta} \Gamma_{\gamma \beta}^{\rho}-\hat{u}_{\rho, \beta} \Gamma_{\gamma \delta}^{\rho}, \\
\hat{u}_{\gamma ; \delta ; \zeta ; \varepsilon ; \beta} \approx \hat{u}_{\gamma, \delta, \zeta, \varepsilon, \beta}-\hat{u}_{\rho, \delta, \zeta, \varepsilon} \Gamma_{\gamma \beta}^{\rho}-\hat{u}_{\gamma, \rho, \zeta, \varepsilon} \Gamma_{\delta \beta}^{\rho}-\hat{u}_{\gamma, \delta, \rho, \varepsilon} \Gamma_{\zeta \beta}^{\rho}-\hat{u}_{\gamma, \delta, \zeta, \rho} \Gamma_{\varepsilon \beta}^{\rho}-\hat{u}_{\rho, \delta, \varepsilon, \beta} \Gamma_{\gamma \zeta}^{\rho}-\hat{u}_{\rho, \zeta, \varepsilon, \beta} \Gamma_{\gamma \delta}^{\rho}- \\
\hat{u}_{\rho, \delta, \zeta, \beta} \Gamma_{\gamma \varepsilon}^{\rho}-\hat{u}_{\gamma, \rho, \varepsilon, \beta} \Gamma_{\delta \zeta}^{\rho}-\hat{u}_{\gamma, \rho, \zeta, \beta} \Gamma_{\delta \varepsilon}^{\rho}-\hat{u}_{\gamma, \delta, \rho, \beta} \Gamma_{\zeta \varepsilon}^{\rho} .
\end{gathered}
$$

Eventually the simplified equation (28) takes the form:

$$
\begin{aligned}
& -c^{\alpha 3 \gamma 3} w_{\gamma, 3,3}+v^{\alpha 3 \gamma 333} w_{\gamma, 3,3,3,3}=\left(c^{\alpha 3 \gamma(\beta)}+c^{\alpha(\beta) \gamma 3}\right) \hat{u}_{\gamma,(\beta), 3}-c^{\alpha \beta \gamma \delta} \Gamma_{\delta \beta}^{3} \hat{u}_{\gamma, 3}-\left(c^{\alpha \beta \gamma 3}+c^{\alpha 3 \gamma \beta}\right) \Gamma_{\gamma \beta}^{\varepsilon} \hat{u}_{\varepsilon, 3}- \\
& 2\left(v^{\alpha 3 \gamma(\beta) 33}+v^{\alpha(\beta) \gamma 333}\right) \hat{u}_{\gamma,(\beta), 3,3,3}+2\left(v^{\alpha \beta \gamma 333} \Gamma_{\gamma \beta}^{\rho}+v^{\alpha 3 \gamma \delta 33} \Gamma_{\gamma \delta}^{\rho}\right) \hat{u}_{\rho, 3,3,3}+ \\
& \left(4 v^{\alpha \beta \gamma \delta 33} \Gamma_{\delta \beta}^{3}+v^{\alpha \beta \gamma 3 \delta 3} \Gamma_{\delta \beta}^{3}+v^{\alpha 3 \gamma \delta 3 \beta} \Gamma_{\delta \beta}^{3}\right) \hat{u}_{\gamma, 3,3,3} .
\end{aligned}
$$

Taking into account that near the body surface the Christoffel symbols and material tensors can be considered as constants, the equation (31) has a very specific form: all of its terms are differentiated with respect to $x^{3}$ at least once. Therefore, there exists a particular solution of this equation obeys an equation with one less derivation:

$$
\begin{aligned}
& -c^{\alpha 3 \gamma 3} w_{\gamma, 3}+v^{\alpha 3 \gamma 333} w_{\gamma, 3,3,3}=\left(c^{\alpha 3 \gamma(\beta)}+c^{\alpha(\beta) \gamma 3}\right) \hat{u}_{\gamma,(\beta)}-c^{\alpha \beta \gamma \delta} \Gamma_{\delta \beta}^{3} \hat{u}_{\gamma}-\left(c^{\alpha \beta \gamma 3}+c^{\alpha 3 \gamma \beta}\right) \Gamma_{\gamma \beta}^{\varepsilon} \hat{u}_{\varepsilon}- \\
& 2\left(v^{\alpha 3 \gamma(\beta) 33}+v^{\alpha(\beta) \gamma 333}\right) \hat{u}_{\gamma,(\beta), 3,3}+2\left(v^{\alpha \beta \gamma 333} \Gamma_{\gamma \beta}^{\rho}+v^{\alpha 3 \gamma \delta 33} \Gamma_{\gamma \delta}^{\rho}\right) \hat{u}_{\rho, 3,3}+ \\
& \left(4 v^{\alpha \beta \gamma \delta 33} \Gamma_{\delta \beta}^{3}+v^{\alpha \beta \gamma 3 \delta 3} \Gamma_{\delta \beta}^{3}+v^{\alpha 3 \gamma \delta 3 \beta} \Gamma_{\delta \beta}^{3}\right) \hat{u}_{\gamma, 3,3} .
\end{aligned}
$$

Note that in the left-hand side of (32) there is exactly the expression that we need in (26). The general solution of the inhomogeneous differential equation is the sum of a particular solution of the inhomogeneous equation and the general solution of the homogeneous equation. But the remarkable fact is that any solution of the homogeneous equation decaying away from the surface, when substituted into $-c^{\alpha 3 \gamma 3} w_{\gamma, 3}+v^{\alpha 3 \gamma 333} w_{\gamma, 3,3,3}$ yields zero. So it is quite enough to consider only a particular solution, and we can simply replace $-c^{\alpha 3 \gamma 3} w_{\gamma, 3}+v^{\alpha 3 \gamma 333} w_{\gamma, 3,3,3}$ in $(26)$ by right-hand side of (32). Having made the replacement, we obtain $s^{\alpha}$ of the form:

$$
\begin{aligned}
& s^{\alpha}=-c^{\alpha 3 \gamma(\delta)} \hat{u}_{\gamma,(\delta)}+c^{\alpha 3 \gamma \delta} \hat{u}_{\varepsilon} \Gamma_{\gamma \delta}^{\varepsilon}+\left(c^{\alpha 3 \gamma(\beta)}+c^{\alpha(\beta) \gamma 3}\right) \hat{u}_{\gamma,(\beta)}-c^{\alpha \beta \gamma \delta} \Gamma_{\delta \beta}^{3} \hat{u}_{\gamma}-\left(c^{\alpha \beta \gamma 3}+c^{\alpha 3 \gamma \beta}\right) \Gamma_{\gamma \beta}^{\varepsilon} \hat{u}_{\varepsilon}- \\
& 2\left(v^{\alpha 3 \gamma(\beta) 33}+v^{\alpha(\beta) \gamma 333}\right) \hat{u}_{\gamma,(\beta), 3,3}+2 v^{\alpha 3 \gamma(\delta) 33} \hat{u}_{\gamma, 3,3,(\delta)}+2 v^{\alpha 3 \gamma 3(\beta) 3} \hat{u}_{\gamma, 3,3,(\beta)}-h^{\alpha \beta} \hat{u}_{\beta, 3,3} .
\end{aligned}
$$

Elementary transformations lead (33) to a rather simple form:

$$
s^{\alpha}=c^{\alpha(\beta) \gamma 3} \hat{u}_{\gamma,(\beta)}-c^{\alpha \beta \gamma \delta} \Gamma_{\delta \beta}^{3} \hat{u}_{\gamma}-c^{\alpha \beta \gamma 3} \Gamma_{\gamma \beta}^{\varepsilon} \hat{u}_{\varepsilon}-h^{\alpha \beta} \hat{u}_{\beta, 3,3},
$$

where $h^{\alpha \beta}$ is redefined as

$$
h^{\alpha \beta}=v^{\alpha(\gamma) \beta 3(\delta) 3} \Gamma_{(\gamma)(\delta)}^{3}-v^{\alpha 3 \gamma 333} \Gamma_{\gamma 3}^{\beta}-2 v^{\alpha 3 \beta \delta 33} \Gamma_{\delta 3}^{3}-v^{\alpha \varepsilon \beta 3 \delta 3} \Gamma_{\delta \varepsilon}^{3} .
$$

This equations together with (18) completely solves the problem under consideration. 


\section{CONCLUSIONS}

In this paper we propose an approach to the description of flexoelectric deformations of finite size bodies with sufficiently smooth surfaces. This approach is based on the fact that in reality the higher elastic moduli are very small. This smallness allows us to represent the elastic displacement vector as a sum of classical and non-classical parts. For the non-classical part the explicit representation derived. As for the classical part, it can be found by solving the equations of the classical theory of elasticity with the boundary conditions obtained in this work. These boundary conditions are also quite classical in the form, so that the classical part of the problem is quite standard and does not require a separate consideration.

1 A.K. Tagantsev, Sov. Phys. Usp. 30, 588 (1987).

2 A.K. Tagantsev, Phase Transit. 35, 119 (1991).

3 P. Zubko, G. Catalan, A.K. Tagantsev, Annu. Rev. Mater. Res. 43, 387 (2013).

${ }_{5}^{4}$ P.V. Yudin, A.K. Tagantsev, Nanotechnology 24, 432001 (2013).

5 A.K. Tagantsev, A.S. Yurkov, J. Appl. Phys. 112, 044103 (2012); arXiv:1110.0380 [cond-mat.mtrl-sci] (2011).

6 A.S. Yurkov, JETP Letters 94, 455 (2011).

7 A.S. Yurkov, arXiv:1304.1868 [cond-mat.mtrl-sci] (2013). 